\title{
ANALYSIS OF THE SPATIOTEMPORAL CHARACTERISTICS OF HEMORRHAGIC FEVER WITH RENAL SYNDROME IN HUBEI PROVINCE, CHINA
}

\author{
Hong Fan, Liang Ge, Liming Song, Qifeng Zhao \\ The State Key Lab. of Information Engineering in Surveying, Mapping, and Remote Sensing(LIESMARS) \\ Wuhan University ,129 Luoyu road Wuhan, China
}

\begin{abstract}
KEY WORDS: Spatiotemporal analysis, Spatiotemporal modelling, Hemorrhagic fever with renal syndrome(HFRS), GIS, Cluster Analysis,
\end{abstract}

\begin{abstract}
:
Hemorrhagic fever with renal syndrome(HFRS) is a worldwide fulminant infectious disease. Since the first HFRS cases in Hubei Province were reported in 1957, the disease has spread across the province and Hubei has become one of seriously affected areas in China. However, the epidemic characteristics of HFRS are still not entirely clear. Therefore, a systematic investigation of spatial and temporal distribution pattern of HFRS system is needed. In order to facilitate better prevention and control of HFRS in Hubei Province, in this paper, a GIS spatiotemporal analysis and modeling tool was developed to analyze the spatiotemporal dynamics of the HFRS epidemic, as well as providinga comprehensive examination the dynamic pattern of HFRS in Hubei over the past 30 years (1980-2009), to determine spatiotemporal change trends and the causes of HFRS. This paper describes the experiments and their results.
\end{abstract}

\section{RELATED WORK}

Hubei Province, is an area that has been severely affected by HFRS for a number of years. HFRS cases in Hubei Province were first reported in a suburb of Wuhan County in 1957. Since then, the disease has spread across the province. In fact, Hubei Province is the area most severely affected by HFRS in the early 1980s(Liu , 2011). HFRS infection has existed in Hubei Province for many years, however, the epidemic characteristics of HFRS are still not entirely clear. Therefore, a systematic investigation of the spatial-temporal distribution pattern of HFRS is needed, in order to facilitate better prevention and control of HFRS in Hubei Province.

There is an extensive literature about the HFRS epidemic in Peoples Republic of China. HFRS was first recognized in 1931 in northeast China, and since 1955, it has appeared in many other places across the nation (Wu , 2009). Currently, HFRS is epidemic in all 31 provinces of the People's Republic of China, and is a significant public health problem with annually 20,00050,000 diagnosed cases (Tang , 2003). In China, HFRS is mainly caused by two types of hantaviruses, the Hantaan virus (HTNV) and the Seoul virus (SEOV), each of which co-evolved within a distinct rodent host. It is also considered human activities and natural factors influent the incidence and prevalence of Hantavirus (Fang 2010). The endemic areas have extended from rural to urban areas, and even into the city center (Zhang, 2004). HFRS incidence has increased in some big cities, such as Beijing, Shenyang, and others (Wu , 2009). This phenomenon has been noted in previous research $(\mathrm{Bi}$, 1998), Bi Peng et al. found that most of the HFRS cases were distributed along river systems such as the Yangtze and the Huai river in Anhui Province.

Although the literature has reported and studied HRFS issues in China, little work has focused on the spatiotemporal distribution and spread patterns of HFRS in China systematically over a long time span.

This current study was designed to use GIS spatial analysis and modeling technologies to investigate the spatiotemporal distribution and spread pattern of HFRS in Hubei Province, China over the past 30 years (1980-2009), and to determine the possible causes.

\section{RESEARCH OVERVIEW}

\subsection{Study Objectives}

The objectives were as follows:

- To develop a spatiotemporal analysis and modeling tool.

- To investigate the spatiotemporal distribution and spreading pattern of HFRS in Hubei Province, China over the past 30 years (1980-2009).

\subsection{The Study Area}

In this paper, we choose the Hubei Province as the research region.

Hubei province (Figure 1) is located in central China (29u05'$\left.33 \mathrm{u} 20^{\prime} \mathrm{N}, 108 \mathrm{u} 21^{\prime}-116 \mathrm{u} 07^{\prime} \mathrm{E}\right)$. It encompasses an area of 189,500 square kilometres and has a population of more than 60 million. Administrative divisions have changed during the past 30 years. In the current study, 76 counties and cities are included in the analyses, as shown in Fig. 1.

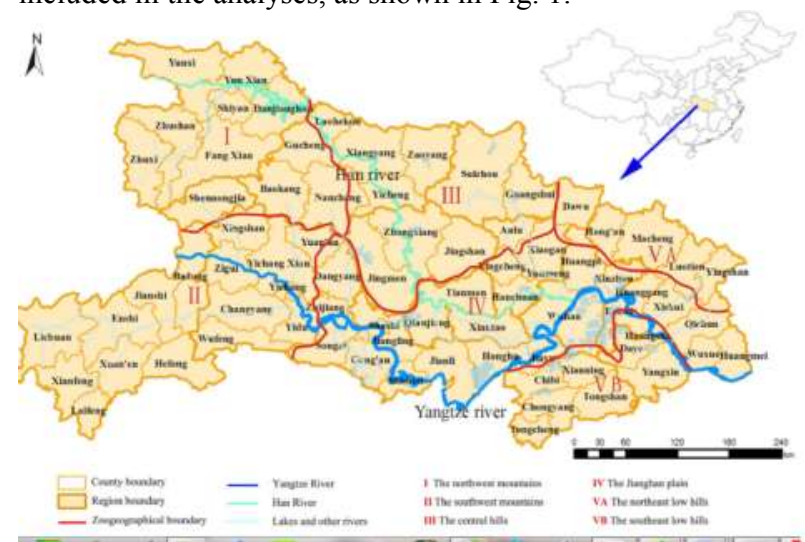

Figure 1. Hubei province and its position in China

\subsection{Data Collection}

Collected data includes: 
- HFRS case: 20000 HFRS disease case in Hubei during 1980 2009, supported by Center for Disease Control and Prevention of Hubei Province (CDCH).

- Hubei county-level vector topographic maps(scale = $1: 4,000,000)$, including the 76 study counties and cities, were used to conduct a GIS-based analysis on the spatial distribution of HFRS. These GIS data were obtained from National Geomatics Center of China(NGCC)

- Demographic data and other statistical data: Population analyses for the periods 1990-1999 and 2000-2009 were based on census records from 1995 and 2000, respectively. obtained from the Hubei Statistics Bureau.

\subsection{Data Processing}

All the data that include the HFRS case data,population data and base map data were used to build geographical information databases and a corresponding attributes database.

The HFRS case data were collected by case number, were firstly processed by county, by month and by year. . All HFRS cases were geocoded according to administrative codes, matched, and associated to the corresponding county/city feature of countylevel polygon map as feature attributes using ArcGIS 10.0.

Population data based on the 1990, 1995, and 2000 censuses was integrated in terms of the administrative code. The 1990 census records were used for population counts of the first decade (1980-1989) studied. Population analyses for the periods 1990-1999 and 2000-2009 were based on census records from 1995 and 2000, respectively.

\subsection{Spatiotemporal Analysis and Modeling Tools}

Spatiotemporal analysis and modelling tools were developed based on ArcGIS server10.0, and brower/server architecture, we choose ArcGIS Server to publish map services as a base map and provide spatial analysis tools. ArcGIS api for flex were used to complete map rendering and build a lightweight, highperformance browser. The tool includes three major function modules: data pre-processing, spatiotemporal analysis tools, and spatiotemporal statistics tools.

\section{SPATIOTEMPORAL ANALYSIS AND MODELING} EXPERIMENT AND RESULTS

\subsection{HFRS Incidence Change Trend Analysis}

In order to better grasp the overall change trends in Hubei, the annual average incidence and fatality of HFRS were calculated for per counties and cities in Hubei, the statistics are shown in Fig. 3. This Figure shows Hubei Province annual HFRS incidence and fatalities normalized by per 10,0000 population.

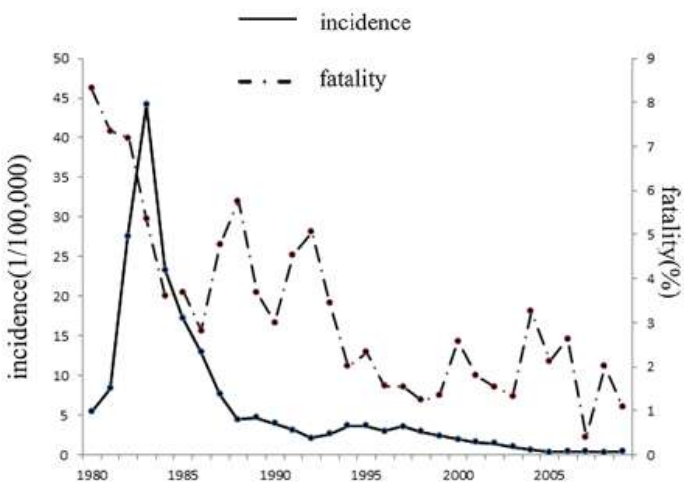

Figure 2. HFRS incidence and fatality trends for each year from 1980 through 2009.

An epidemic curve (Figure 2) shows that a total of 104,467 HFRS cases were reported in Hubei between 1980 and 2009, a peak appeared in the 80s. In 1983 the incidence exceeded 30/100,000. After 1990 the incident decreased overall, in 2003, annual incidence of HFRS gradually decreased and has drooped to below $1 / 100,000$.

As can be seen from Figure 3, the highest rate of fatalities due to HFRS was $8.31 \%$, the lowest yearly rate of fatalities due to HFRS was $0.4 \%$ occurring in 2007 . The rate of fatalities due to HFRS decreased after 1980 sharply and has remained at approximately $5 \%$, followed by a slight decline in fatalities. Over the past decade the rate of fatalities due to HFRS fluctuated up and down around $2 \%$ reducing to its lowest rate in 2007( 0.4\%).

\subsection{Seasonal analysis}

To determine how HFRS incidents are influenced by seasonal change, monthly HFRS incidences in Hubei Province over the 30 year study period were calculated(Fig. 3 ).

From Fig. 3, we can conclude:

In the early 1980s a single epidemic peak occurred in the fallwinter season, from October to February of the coming year, occupy more than $80 \%$ incidences for the whole year. In the mid-1980s to the mid-1990s, the incidence of the fall-winter season still dominated, but the proportion of incidents slightly declined. In the 1990's, the seasonal epidemic pattern changed significantly from the 1980 s, a small epidemic peak between April and August appeared, and after 1995, HRFS displayed a prominent bi-peak epidemic pattern.

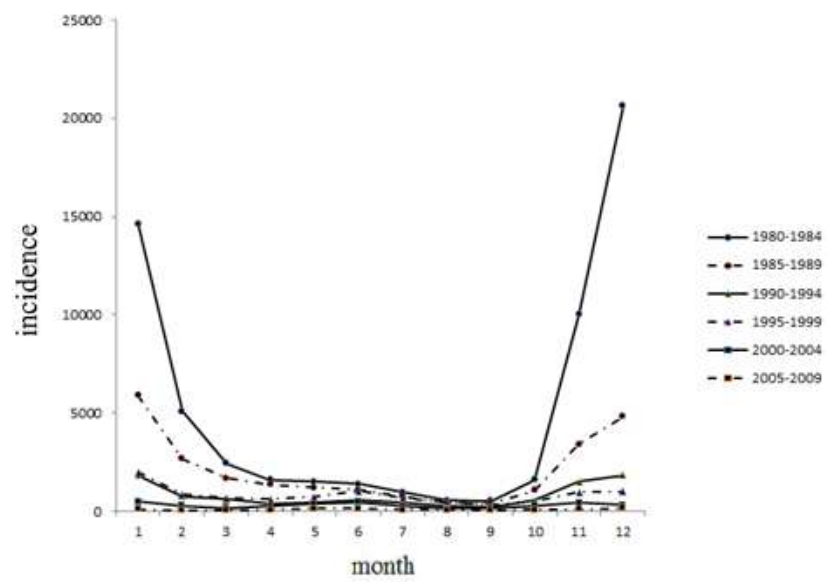

Figure 3. Hubei monthly incidents of HRFS for every five year from 1980 through 2009. 


\subsection{Spatial distribution of HFRS}
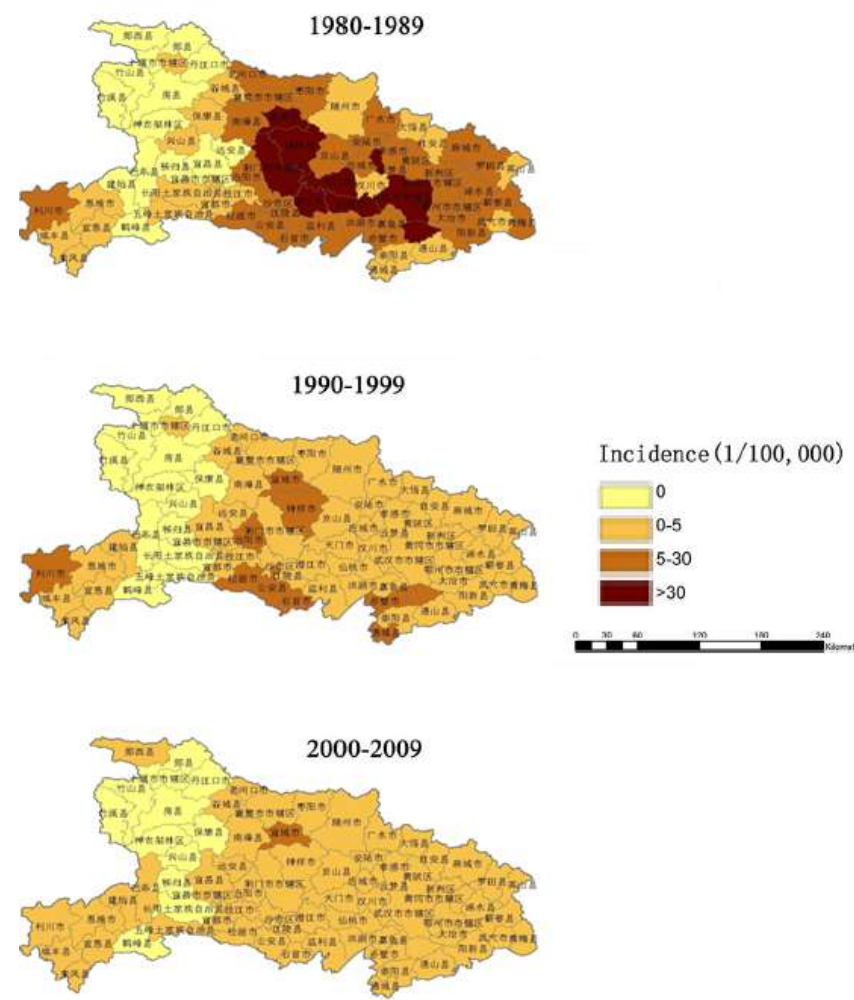

Figure 4 Hubei HFRS Spatial Distribution every ten years from 1980 through 2009.

Use ten years as a division, according to the national unified HFRS monitoring criteria, the epidemic strength of the city and county was divided into four classes, high incidence (incidence $>30 / 10$ million), middle (incidence of 5 to $30 /$ 100,000 ), and low development zone (incidence $<5 / 10$ million) and non-infected (incidence 0 ). the incidence distribution of HFRS in Hubei Province from 1980 to 1989,1990 to 1999 , from 2000 to 2009 were mapped and are shown in Fig 4.

It can be seen from Fig. 4 that for every ten year period, the incidence distribution, in the 1980s, high HFRS incidence include nine counties: Yicheng, Zhongxiang, Jingmen, Wuhan and et. al., located in the central Hubei Province, other area are in the middle incident zone; in the 1990s the middle incident zones were mainly distributed in the central and southern counties of Hubei province; after 2000, only a few cities and counties were the middle incident zone, the rest were lowincident zones and non-infected zones. The epidemic area however, has expanded in comparison with the 1980s, the southwestern counties have become the new epidemic area.

\subsection{Space-time clustering analysis}

Hubei HFRS incidences, demographic, and geographic data are used as the data source of Space-time clustering analysis;a Poisson function is adopted to implement spatiotemporal clustering analysis of HFRS incidences in Hubei. In detail, during the clustering process, the maximum window radius is set to less than $25 \%$ of the total population; and the time step is set to a year.

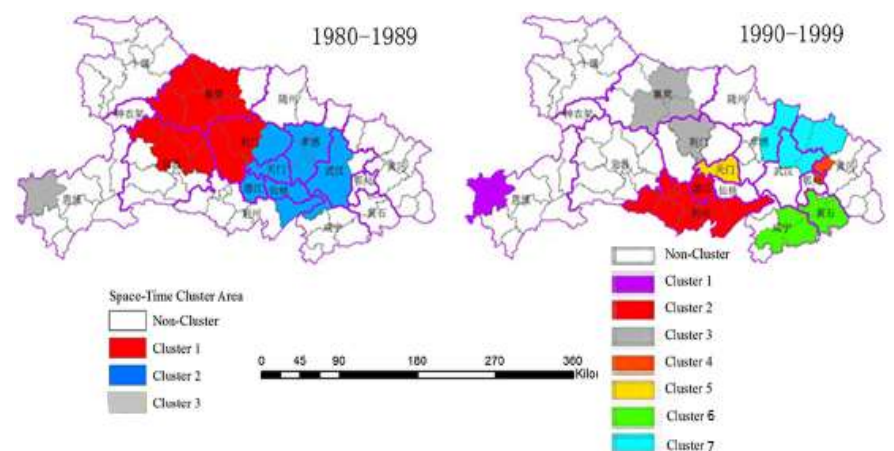

Figure5. Hubei HFRS Spatio-temporal Cluster Results for 80 89,90 99

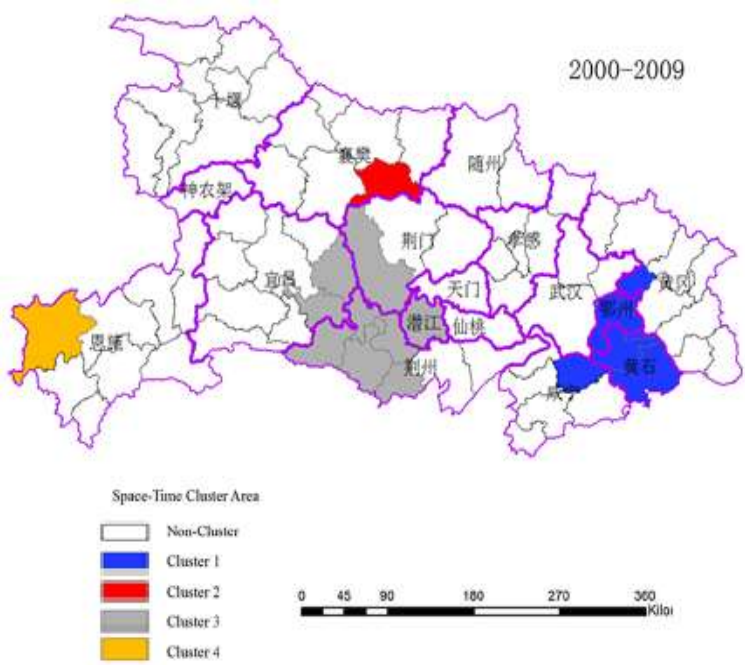

Figure6. Hubei HFRS Spatio-temporal Cluster Results for $00 \sim 09$

\begin{tabular}{|c|c|c|c|c|c|c|c|c|}
\hline $\begin{array}{c}\text { Timeperio } \\
\mathrm{d}\end{array}$ & Cluster: & Start & End & LLR & P & $\begin{array}{c}\text { Observe } \\
\text { dCaseNu } \\
\text { mber }\end{array}$ & $\begin{array}{c}\text { Expecte } \\
\text { dCaseN } \\
\text { umber }\end{array}$ & $R R$ \\
\hline \multirow{3}{*}{$1980 \sim 1989$} & 1 & 1982 & 1983 & 25978.42 & $<0.001$ & 24330 & 4072.30 & 794 \\
\hline & 2 & 1983 & 1984 & 2493.62 & $<0.001$ & 6361 & 2309.17 & 290 \\
\hline & 3 & 1981 & 1982 & 7278 & $<0.001$ & 491 & 270.16 & 1.82 \\
\hline \multirow{7}{*}{$1990 \sim 1999$} & 1 & 1991 & 1992 & 2071.54 & $<0.001$ & 1022 & 53.47 & 20.12 \\
\hline & 2 & 1995 & 1996 & 84593 & $<0.001$ & 1614 & 485.05 & 3.54 \\
\hline & 3 & 1993 & 1994 & 50529 & $<0.001$ & 956 & 28196 & 351 \\
\hline & 4 & 1998 & 1999 & 35498 & $<0.001$ & 327 & 4723 & 7.03 \\
\hline & 5 & 1990 & 1991 & 293.04 & $<0.001$ & 456 & 114.06 & 4.07 \\
\hline & 6 & 1990 & 1991 & 176.01 & $<0.001$ & 541 & 215.01 & 256 \\
\hline & $?$ & 1997 & 1997 & 531 & 0.893 & 241 & 194.12 & 1.24 \\
\hline \multirow{6}{*}{$2000 \sim 2009$} & 1 & 2000 & 2001 & 509.81 & $<0.001$ & 494 & 78.86 & 6.86 \\
\hline & 2 & 2002 & 2003 & 37291 & $<0.001$ & 182 & 9.23 & 20.44 \\
\hline & 3 & 2000 & 2001 & 319.81 & $<0.001$ & 483 & 1213 & 431 \\
\hline & 4 & 2000 & 2001 & 155.09 & $<0.001$ & 118 & 13.16 & 9.16 \\
\hline & 5 & 2001 & 2001 & 154 & 1 & 10 & 5.43 & 1.84 \\
\hline & 6 & 2000 & 2000 & 0.23 & 1 & 72 & 66.48 & 1.08 \\
\hline
\end{tabular}

Table 1. Detection of HFRS Clusters in Hubei Province, China

The results of spatial scans are shown in Table 1, Fig. 5 and Fig 6. It can be seen from 1980 to 1989, one highest incidence cluster and two secondary clusters are statistically significant. During the period from 1990 to 1999, one highest incidence cluster and five secondary cluster areas are observed, During the period from 2000 to 2009 , one highest incidence cluster area is observed with 7.94 relative risk. Among them, from 1980 to 1989, the highest incidence cluster area included HanChuan,Yincheng, Yunmeng, Xiantao, Tianmen, the Wuhan, Xiaogan, Honghu, Jiayu, Jingshang, Anlu, Huangpi,and Qianjiang. From 1990 to 1999 , the highest incidence cluster include Lichuang, and its relative risk is 20.12. From 2000 to 
2009, the highest incidence cluster include Daye, Huangshi, Echeng, Yangxin, Xianning and Huanggang.

\section{CONCLUSION}

HFRS has been a serious concern in Hubei for many decades. In the current study, with the help of GIS spatio-temporal analysis and modeling tools we developed, we describe the spatiotemporal distribution and seasonal patterns of HFRS in Hubei Province over a 30-year epidemic period (1980-2009). The HFRS incidence has displayed different epidemic characteristics over the past three decades. The annual incidence and fatality rates due to HFRS have declined since the outbreaks in the 1980's. HFRS incidence has become highly sporadic in recent years. We found that many districts that contain or that are close to large lakes or rivers, such as Lichuan, Zhongxiang, Jingmen, Songzi, Gong'an, Shishou, Honghu, Qianjiang and Wuhan, have high incidences of HFRS during certain times. The geographical distribution of HFRS in Hubei suggests that environmental factors, such as terrain and water may play important roles in supporting rodent populations, increasing the risk of HFRS in certain areas of Hubei.

A notable shift from a single-peak to a bi-peak epidemic pattern was observed in our seasonal analysis. During the last decade, the HFRS has begun to occur more frequently in the spring and summer, accounting for about $50 \%$ of all cases. This is consistent with the changing trends of other epidemic disease patterns in Mainland China, where many endemic areas that were dominated by HTNV have become bimodal pattern endemic areas or SEOV dominant (Reusken , 2013). It is the rapid socioeconomic development causing the wide dispersion of Brown Norway rats and the emergence of new Seoul variants that might subsequently lead to increase in the prevalence of SEOV infections in Hubei.

The GIS spatiotemporal analysis and modeling tools described in this paper can help domain experts to uncover s unknown facts and information hidden in the data. The mechanisms that drive the changing epidemiology of HFRS are complex and multifactorial (Bi , 2003), and require further discussion and analysis with the cooperation of both domain experts and GIS Experts.

\section{ACKNOWLEDGEMENTS (OPTIONAL)}

This work was supported by grants from the National Natural Science foundation Projects of China ( No. 41471323, PI: Dr. Hong Fan, No. 41023001, PI: Dr. Jianya Gong)

\section{REFERENCES}

Wu W, Guo JQ, Yin ZH, Wang P, Zhou BS (2009) GIS-based spatial, temporal and space-time analysis of haemorrhagic fever with renal syndrome. Epidemiology and Infection 137: 1766.

Fang LQ, Wang XJ, Liang S, Li YL, Song SX, et al. (2010) Spatiotemporal trends and climatic factors of hemorrhagic fever with renal syndrome epidemic in Shandong Province, China. PLoS Negl Trop Dis 4: e789.

Bi P, Tong S, Donald K, Parton K, Ni J (2002) Climatic, reservoir and occupational variables and the transmission of haemorrhagic fever with renal syndrome in China. Int $\mathrm{J}$ Epidemiol 31: 189-193.

Zhang YZ, Xiao DL, Wang Y, et al. (2004) The epidemic characteristics and preventive measures of hemorrhagic fever with syndromes in China. Zhonghua Liu Xing Bing Xue Za Zhi [in Chinese] 25: 466-469.

Tang F, Cao WC (2003) Ecological and epidemiological studies on rodent hosts of hantavirus. Chin J Vector Bio\&Control 14: 315-317.

Bi P, Wu X, Zhang F, Parton KA, Tong S (1998) Seasonal rainfall variability, the incidence of hemorrhagic fever with renal syndrome, and prediction of the disease in low-lying areas of China. Am J Epidemiol 148: 276-281.

Chen HX, Qiu FX (1993) Epidemiologic surveillance on the hemorrhagic fever with renal syndrome in China. Chinese medical journal 106: 857-863.

Chen HX, Qiu FX, Zhao XQ, Luo CW, Li XQ (1994) Characteristics of the Distribution of Epidemic Season of Hemorrhagic Fever with Renal Syndrome in Different Regions and Different Years in China. Zhonghua Liu Xing Bing Xue Za Zhi [in Chinese] 8: 197-203.

Reusken C, Heyman P (2013) Factors driving hantavirus emergence in Europe. Current Opinion in Virology 3: 92-99.

Liu X, Jiang B, Gu W, Liu Q (2011) Temporal trend and climate factors of hemorrhagic fever with renal syndrome epidemic in Shenyang City, China. BMC Infectious Diseases 11: 331.

Zuo SQ, Fang LQ, Zhan L, Zhang PH, Jiang JF, et al. (2011) Geo-spatial Hotspots of Hemorrhagic Fever with Renal Syndrome and Genetic Characterization of Seoul Variants in Beijing, China. PLoS Neglected Tropical Diseases 5: e945.

Xiao SY, Liang M, Schmaljohn CS (1993) Molecular and antigenic characterization of HV114, a hantavirus isolated from a patient with haemorrhagic fever with renal syndrome in China. J Gen Virol 74 (Pt8): 1657-1659.

Tang F, Cao WC (2003) Ecological and epidemiological studies on rodent hosts of hantavirus. Chin J Vector Bio\&Control 14: 315-317. 